\title{
Global, Nonaxisymmetric Instabilities in Magnetized Accretion Disks
}

\author{
Charles Curry ${ }^{1}$ and Ralph E. Pudritz \\ Department of Physics and Astronomy, McMaster University, \\ Hamilton, ON L8S 4M1, Canada
}

\begin{abstract}
We investigate the global stability of a differentially rotating, ideal MHD fluid shell to linear, nonaxisymmetric perturbations. This system, which approximates an accretion disk near its midplane, is known to be unstable to both axisymmetric and nonaxisymmetric local perturbations. Keplerian disks, which are entirely stable in the hydrodynamic case, show the most rapid growth for radially extended shells.
\end{abstract}

\section{Introduction}

Over a decade ago, Papaloizou \& Pringle (1984) (hereafter PP) identified a global, hydrodynamic, nonaxisymmetric instability of a differentially rotating torus whose dynamical growth pointed to interesting consequences for the structure of accretion disks. More recently, Balbus \& Hawley (1992) (hereafter BH) found a nonaxisymmetric, local instability of magnetized accretion disks, with a comparable growth time. Since the large-scale effects of the latter instability, however, cannot be ascertained from the local analysis, we present here the results of a global (in the radial direction) linear stability calculation. We refer the interested reader to Curry \& Pudritz (1996) (hereafter CP) for details which are for the sake of brevity omitted here.

\section{Equilibria and Perturbations}

A global analysis requires consideration of the boundaries of the disk. Here we model the effect of radial boundaries only. Consider a cylindrical shell of incompressible fluid of infinite extent in the $z$-direction, rotating about the $z$ axis. A uniform axial magnetic field $\mathbf{B}=B \hat{\mathbf{z}}$ threads the fluid and the surrounding vacuum; the former is assumed to be sufficiently ionized so that the equations of ideal MHD apply. Self-gravity is ignored, but a central object at $r=z=0$ generates the potential $\Psi=-G M / r$. Taking a rotation law of the form $\Omega(r) \propto r^{-a}, 3 / 2 \leq a \leq 2$, the inner and outer radii of the fluid, $r_{1}$ and $r_{2}$, are uniquely specified by the rotation index $a$. Equilibrium is maintained by a balance of centifugal forces, pressure gradients, and the central gravity.

\footnotetext{
${ }^{1}$ Present address: Dept. of Astronomy, University of California, Berkeley, CA, USA 94720.
} 


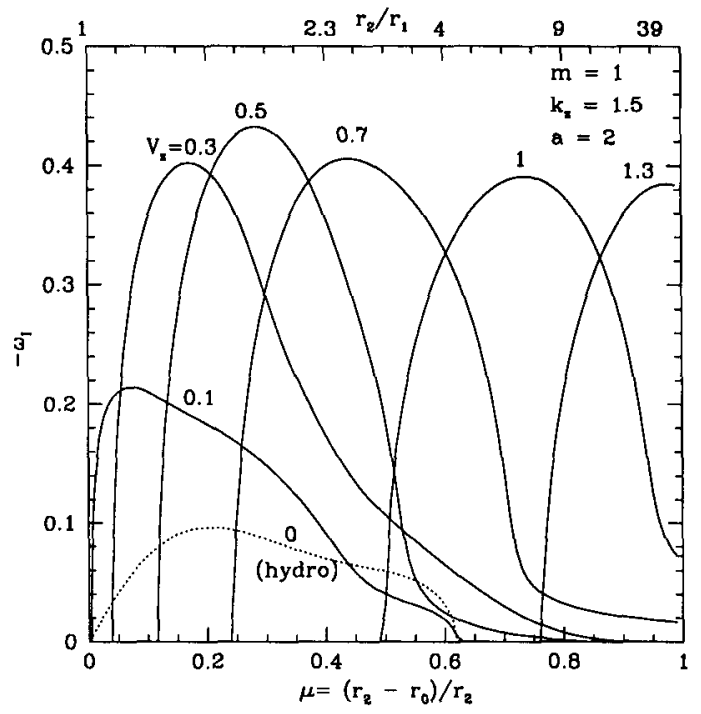

Figure 1. Growth rates of the $m \neq 0$ MHD instability as a function of cylinder thickness ( $\mu$, bottom; $r_{2} / r_{1}$, top) for a range of $V_{z}$. The hydrodynamic case is given by the dotted line.

Assuming perturbations of the form $\delta X=\delta X(r) \exp \left[i\left(m \phi+k_{z} z+\omega t\right)\right]$ where $X$ is any physical variable, linearization of the equilibrium equations and some manipulation leads to a second-order ordinary ODE for the perturbed radial velocity. This equation was solved numerically as an eigenvalue problem for $\omega$, subject to free boundary conditions (CP). Unstable, growing modes (i.e. $\left.\omega=\omega_{R}+i \omega_{I}, \omega_{I}<0\right)$, were found and are discussed below.

\section{Results}

Figure 1 shows the growth rate, $-\omega_{I}$, as a function of the shell thickness parameter, $\mu$, for a range of magnetic field strengths, characterized by the Alfvén speed, $V_{z} \equiv B / \sqrt{4 \pi \rho}$. The rotation law is that of constant angular momentum $(a=2)$. Note that units of length and time have been scaled to the pressure maximum values $r_{0}, \Omega_{0}^{-1}$. Some notable features are: (1) The magnetic field stabilizes for thicknesses $\mu<\mu_{\text {min }}$, where $\mu_{\min }$ increases with $V_{z}$. (2) For stronger fields $\left(V_{z}>0.15\right)$, even the most extended shells are unstable, in contrast to the hydrodynamic case (Blaes \& Glatzel 1986). (3) Each configuration of specified $\mu$ possesses a critical field strength $V_{z, \text { crit }}$ above which it is stable.

The unstable modes represented in Fig. 1 are actually hybrids of two distinct modes, which we label $\mathcal{P}$ (for PP mode) and $\mathcal{A}^{-}$(for slow Alfvénic mode), denoting the hybrid by $\mathcal{P} / \mathcal{A}^{-}$. We also found a fast Alfvénic mode, $\mathcal{A}^{+}$, and the resulting hybrid $\mathcal{P} / \mathcal{A}^{+}$. The circumstances determining which of these modes are present can be seen from Figure 2, which is identical to Fig. 1 except that it displays a range of rotation laws $a$ for fixed $V_{z}=0.3$. 

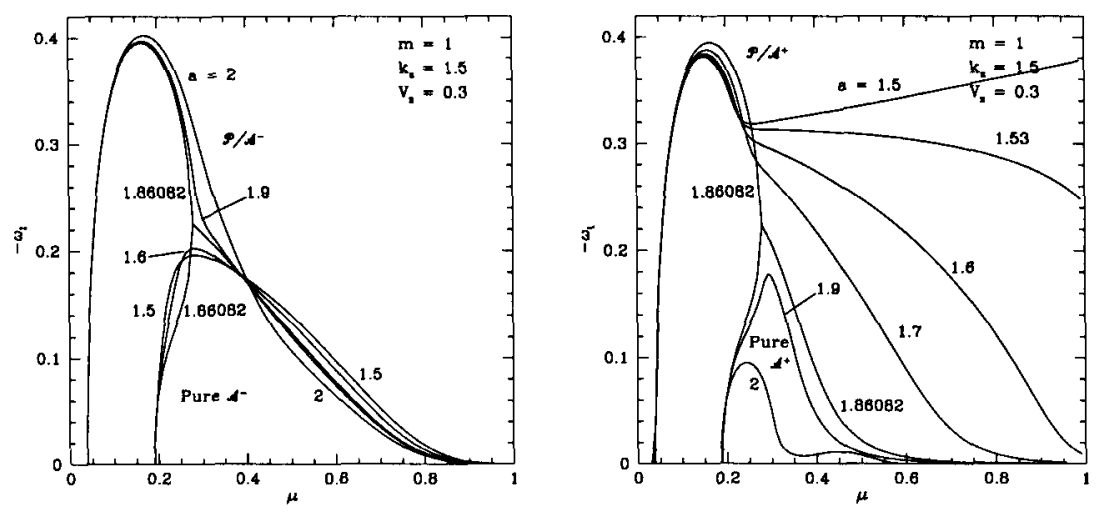

Figure 2. The effect of rotation law on growth rates of the (a) $\mathcal{P} / \mathcal{A}^{-}, \mathcal{A}^{-}$, (b) $\mathcal{P} / \mathcal{A}^{+}$and $\mathcal{A}^{+}$modes.

For thin shells, $\mu<0.2$, the instability is of a purely hydrodynamic and surface (or $\mathcal{P}$ ) nature, while more extended shells are susceptible to two separate instabilities; one which is a hybrid of $\mathcal{P}$ and $\mathcal{A}^{-}$or $\mathcal{A}^{+}$, and another which is purely $\mathcal{A}^{-}$or $\mathcal{A}^{+}$. The critical curve $a_{c}=1.86082 \ldots$ acts as a separatrix between the pure and hybrid behaviors (but note that $a_{c}$ depends on $k_{z}$ and $V_{z}$ ). The large growth rate of the $\mathcal{P} / \mathcal{A}^{+}$mode for near-Keplerian rotation laws is particularly intruiging, since the corresponding growth rate in the hydrodynamic case is entirely negligible (Jaroszyński 1988).

We posit that the mechanism of the instability in large-thickness shells is the over-reflection of internal waves, a well-studied process in purely hydrodynamic flows (e.g. Narayan \& Goodman 1989). The principal difference in the present case is the diminished importance of the corotation radius, $r_{C R}=\left(-\omega_{R} / m\right)^{-1 / a}$, compared to the inner and outer Alfvén radii, $r_{A, \mp}=\left[-\left(\omega_{R} \mp k_{z} V_{z}\right) / m\right]^{-1 / a}$ (the latter are singularities of the MHD perturbation equation; $r_{C R}$ is not). The location of $r_{A, \mp}$ with respect to $r_{1}$ and $r_{2}$ determines whether internal Alfvén waves are amplified or damped (CP).

Finally, we investigated the dependence of feature (3) above on $k_{z}, V_{z}$, and $a$. Considering $V_{z}$ as a function of $\mu_{\min }$, the former represents the critical field strength above which a shell of radial thickness $\mu_{\min }$ is stable to $m \neq 0$ perturbations (Curry, Pudritz \& Sutherland 1994, hereafter CPS; CP). Figure 3 shows the dependence of $V_{z}$ on $\mu_{\min }$ for four different $k_{z}, a=1.5$ and $a=2$. Models lying above a given curve are stable to the $m=1$ MHD instability. Following CPS, we interpret the inverse of the wavenumber $\lambda=2 \pi / k_{z}$ as a critical wavelength above which unstable perturbations no longer fit inside a disk of 'simulated scaleheight' $H=\lambda / 2$. The scaleheights so defined also appear on the graph. Comparison with the $m=0$ results of CPS shows qualitative agreement between the $m=1$ and $m=0$ critical stability curves. However, disks large in both radial (large $\mu_{\min }$ ) and vertical (small $k_{z}$ ) dimension appear more unstable to $m \neq 0$ modes than to $m=0$ ones. 


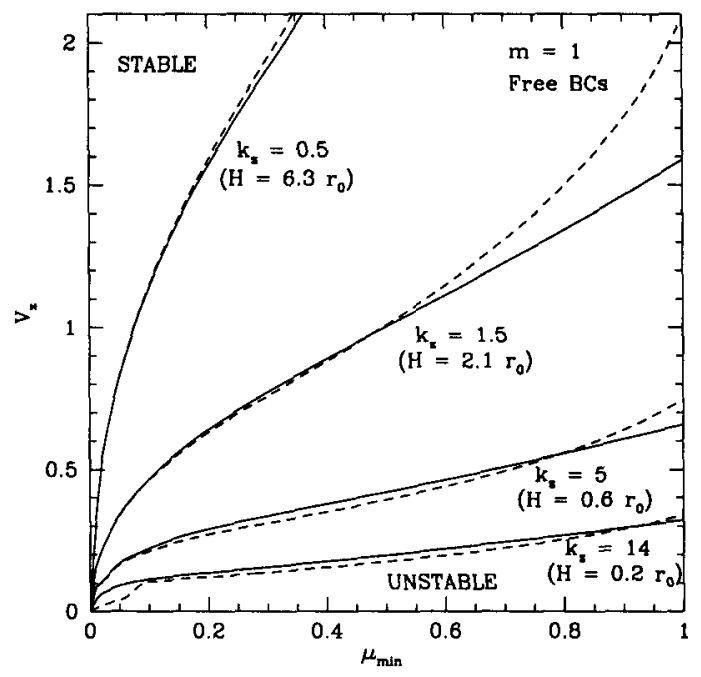

Figure 3. Critical Alfvén speed, $V_{z}$, as a function of $\mu_{\min }$ for $m=1$. Curves are labelled by $k_{z}$ and the corresponding scaleheight $H$. The solid curve is for $a=2$; dashed curve for $a=1.5$.

\section{Conclusions}

We have demonstrated the existence of globally unstable, nonaxisymmetric modes in incompressible MHD cylinders and, by extension, astrophysical disks. The PP instability is enhanced by the presence of a magnetic field, and entirely new unstable modes appear. The most rapidly-growing $\left(\mathcal{P} / \mathcal{A}^{+}\right)$modes occur for the most extended configurations with Keplerian rotation laws, and feature $-\omega_{I}$ in excess of $\Omega$ (but do not exceed the local axisymmetric growth rates). This result alone may have important implications for angular momentum transport and the generation of large-scale ordered fields in accretion disks.

\section{References}

Balbus S. A., \& Hawley J. F., 1992, ApJ, 400, 610 (BH)

Blaes O., \& Glatzel W., 1986, MNRAS, 220, 253

Curry, C., \& Pudritz, R. E. 1996, MNRAS, 281, 119

Curry, C., Pudritz, R. E., \& Sutherland, P. G. 1994, ApJ, 434, 206 (CPS)

Hawley, J. F., \& Balbus, S. A. 1991, ApJ, 376, 233

Jaroszyński M., 1988, Acta Astron., 38, 289

Narayan R., \& Goodman J., 1989, in Meyer F., Duschl W. J., Frank J.,MeyerHofmeister E., eds, Theory of Accretion Disks. Kluwer, Dordrecht, p. 231

Papaloizou J. C. B., \& Pringle J. E., 1984, MNRAS, 208, 721 (PP) 


\section{Discussion}

S. Balbus: Do I understand that in the absence of a reflecting boundary, you would predict stability for the disk? That is odd, because the weak-field instability is triggered locally.

C. Curry: No. Although I suspect that non-reflecting boundaries would reduce the growth rate of the internal Alfvén modes, they will still be locally unstable. Also, as long as the boundaries remain free, the unstable surface mode will still be present.

U. Torkelsson: What are the radial boundary conditions?

C. Curry: The radial boundary conditions are that the normal component of the Lagrangian stress and the magnetic flux are continuous across the boundaries. 